\title{
Feasibility and safety of robotic resection of complicated diverticular disease
}

\author{
Fabian Grass $^{1}$ [D · Jacopo Crippa ${ }^{1} \cdot$ Kellie L. Mathis ${ }^{1} \cdot$ Scott R. Kelley ${ }^{1} \cdot$ David W. Larson ${ }^{1}$
}

Received: 17 October 2018 / Accepted: 1 March 2019 / Published online: 13 March 2019

(c) Springer Science+Business Media, LLC, part of Springer Nature 2019

\begin{abstract}
This study aimed to assess intra- and postoperative outcomes of robotic resection of left-sided complicated diverticular disease. Retrospective analysis of a prospectively maintained institutional database on consecutive patients undergoing elective robotic resection for diverticular disease (2014-2018). All procedures were performed within an enhanced recovery pathway (ERP). Demographic, surgical and ERP-related items were compared between patients with simple and complicated diverticular disease according to intra-operative presentation. Postoperative complications and length of stay were compared between the two groups. Out of 150 patients, 78 (52\%) presented with complicated and the remaining 72 (48\%) with uncomplicated disease. Both groups were comparable regarding demographic baseline characteristics and overall ERP compliance. Surgery for complicated disease was longer ( $288 \pm 96$ vs. $258 \pm 72 \mathrm{~min}, p=0.04)$ and more contaminated ( $\geq$ class $3: 57.7$ vs. $23.6 \%, p<0.001)$ with a trend to higher conversion rates $(10.3$ vs. $2.8 \%, p=0.1)$. While postoperative overall complications tended to occur more often after resections for complicated disease ( 28.2 vs. $15.3 \%, p=0.075)$, major, surgical and medical complications did not differ between the two groups, and median length of stay was 3 days in both settings $(p=0.19)$. Robotic resection of diverticular disease was feasible and safe regardless of disease presentation by the time of surgery.
\end{abstract}

Keywords Robotic $\cdot$ Diverticular disease $\cdot$ Complications $\cdot$ Enhanced recovery

Diverticular disease remains a major health issue in Western societies, and surgical management due to recurrent or complicated disease flares remains inevitable in many patients [1]. A recent randomized controlled trial comparing surgical to conservative management found superior quality of life in surgically managed patients with recurrent and persistent abdominal complaints [2]. However, a 6-year national observational study revealed that surgical outcomes after elective colectomy for diverticular disease was worse compared to patients with colon cancer, underlining the complexity of surgical management in these patients [3].

Due to its potential to overcome technical limitations of laparoscopy in deep and narrow anatomic spaces, robotic surgery emerged as a technical alternative in recent years [4]. However, a recently published landmark trial failed to demonstrate benefits in terms of conversion or complication rates in rectal cancer surgery [5].

David W. Larson

larson.david2@mayo.edu

1 Division of Colon and Rectal Surgery, Mayo Clinic, 200

First Street SW, Rochester, MN 55905, USA
While initial adoption of a robotic approach demands financial and technical efforts, more experienced centers have expanded their indications and emphasize the advantages of better exposure, increased dexterity and a steady visual field [6]. Our institution has progressively increased the use of the robotic platform after observing encouraging results in oncologic and pouch surgery [7-9]. As a consequence, indications have expanded to include complex diverticular disease, through with limited data regarding outcomes.

The present study aims to analyze short-term outcomes after elective robotic resection of complicated left-sided diverticular disease in patients treated within a comprehensive enhanced recovery pathway (ERP).

\section{Materials and methods}

All consecutive patients undergoing elective robotic leftsided or sigmoid colonic resection for diverticular disease at our institution were included in this retrospective analysis (January 2014-May 2018). Data were derived from a 
prospectively maintained institutional database. The study was approved by the institutional review board and written patient consent was obtained to analyze de-identified data. Included patients were assigned to two groups (uncomplicated vs. complicated diverticular disease) according to disease presentation at the time of surgery, previous disease course, and intraoperative and pathological findings.

Mandatory for assigning a patient to either group was disease presentation at the time of surgery according to imaging findings (computed tomography scans, evaluated by boardcertified radiologists), which were correlated with intraoperative presentation and pathological reports. Complicated diverticular disease was defined as phlegmonous, pyogenic disease with presence of an abscess of $>1 \mathrm{~cm}$, fistulizing disease (colo-vesical, -vaginal, -enteric) or contained perforation during surgery. Uncomplicated disease was defined as chronic or recurrent smoldering disease with none of the aforementioned findings and according to institutional definitions [10]. Since the present study focused on surgical feasibility, previous disease course was less important unless a clinically apparent fistula was present pre-operatively to indicate a priori complicated disease.

Demographic characteristics included age, gender, body mass index (BMI), American Society of Anesthesiology (ASA) score and immunosuppression within 2 weeks of surgery. Surgical details included case duration, conversion to open approach, wound class [11], additional organ resection during the same procedure, creation of either a stapled anastomosis or end ostomy and use of a temporary diversion (loop ileostomy). Postoperative hemoglobin levels ( $\mathrm{g} / \mathrm{dL}$ ) were measured at postoperative day (POD) 1 .

\section{Procedure and perioperative care}

All surgeries were performed by 4 experienced board-certified colorectal surgeons through a standardized approach using the Da Vinci® Xi platform. All participating surgeons had performed at least 20 robotic colorectal resections before operating on complicated diverticular cases. All surgeons were either performing the procedure by themselves, or assisting colorectal fellows in the setting of our teaching institution under direct supervision.

Briefly, to access the abdominal cavity, an Optiview ${ }^{\circledR}$ trocar (Ethicon Endo-Surgery, Inc.) was placed under direct visualization in the midline, either superior or inferior to the umbilicus, and approximately $15 \mathrm{~cm}$ away from the target anatomy. Once the trocar was in place and the abdomen insufflated, a 30 degree down angle camera was introduced with placement of 4 additional trocars under direct visualization. To decrease issues with internal and external collisions, trocars were placed at a distance of $6-8 \mathrm{~cm}$ across the abdomen in a horizontal line with the camera. For specimen extraction, a small Pfannenstiel incision $2 \mathrm{~cm}$ above the pubis was created.

All patients were treated within a comprehensive ERP, which has been implemented for colorectal surgery in 2010 [12] and focused on

- perioperative fluid restriction with saline lock at 08:00 h on POD 1

- early removal of urinary catheter at 08:00 h on POD 1

- multimodal pain management including postoperative non-steroidal anti-inflammatory drug (NSAIDs) administration

- promotion of early mobilization and re-introduction of a normal diet within $48 \mathrm{~h}$ of surgery

Overall ERP compliance and compliance to specific postoperative recovery targets was compared between the two groups.

\section{Outcome}

The primary outcome was overall complication rate, classified according to the Dindo-Clavien grading scale as grade I-V [13]. Complications were further stratified in minor (I-IIIa), major (IIIb-V), surgical and medical. Specific surgical complications included postoperative ileus (defined as need for nasogastric tube reinsertion), small bowel obstruction (SBO), clinically or radiographically confirmed anastomotic leak, surgical site infection (SSI), bleeding complications (defined as need for peri- or postoperative transfusion) and urinary retention (defined as the need for urinary catheter re-insertion).

Reoperation, readmission, and length of stay were assessed. All complications were in-hospital and until 30 days postoperatively.

\section{Statistical analysis}

Descriptive statistics for categorical variables were reported as frequency (\%), continuous variables as mean (standard deviation) or median (interquartile range (IQR)) according to distribution. $\chi^{2}$ test was used to compare categorical variables. All statistical tests were two-sided and a level of $\leq 0.05$ was used to indicate statistical significance. Since both study groups were comparable, statistical matching was not necessary. Data analysis was performed with the Statistical Software for the Social Sciences SPSS Advanced Statistics 22 (IBM Software Group, 200 W. Madison St., Chicago, IL; 60,606 USA). 


\section{Results}

One hundred and fifty patients underwent robotic leftsided or sigmoid resection over the study period. Of these, $78(52 \%)$ presented with complicated and $72(48 \%)$ with uncomplicated disease. Disease presentation for complicated disease was as follows:

- phlegmonous, pyogenic disease in 54 cases $(69.2 \%)$

- fistulizing disease in 33 patients (42.3\%)

- contained perforating disease in 11 patients (14.1\%)

Both groups were comparable regarding demographic characteristics (Table 1).

Surgery for complicated disease was longer and more contaminated with a trend to higher conversion rates (10.3 vs. $2.8 \%, p=0.1$ ), and temporary diversion was more often performed ( 29.5 vs. $13.9 \%, p=0.029$, Table 1 ). Additional organ resections, performed in 17 patients (11.3\%), were salpingo-oophorectomies $(x 5)$, small bowel resections $(x 4)$, appendectomies $(x 4)$, hysterectomies $(x 3)$ and partial bladder resection $(x 1)$. Overall, 28 patients $(18.7 \%)$ had previous abdominal surgery, and adhesiolysis of at least 30 min was performed in 12 of these patients, with equal distribution among the 2 groups ( 6 patients (7.7\%) vs. 6 patients (8.3\%), $p=0.89$ ).

Overall compliance to the institutional ERP was comparable between both groups. Compliance to specific postoperative items is illustrated in Fig. 1.

Postoperative overall complications after resections for complicated disease $(28.2$ vs. $15.3 \%, p=0.075)$ were no

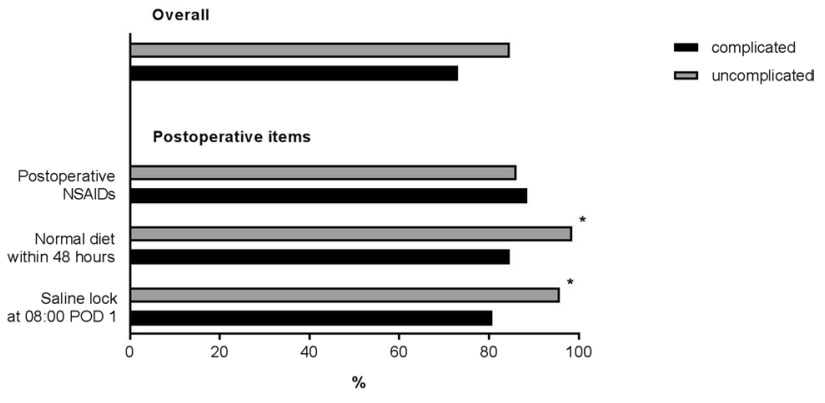

Fig. 1 ERP compliance. ERP overall compliance and compliance to selected postoperative items of patients with complicated diverticulitis $(n=78)$ and patients with uncomplicated diverticulitis $(n=72)$. NSAID non-steroidal anti-inflammatory drug, $P O D$ postoperative day Asterisk indicate statistical significance $(p<0.05)$

different; moreover, major, surgical and medical complications did not differ between the two groups (Table 2). Overall, five patients $(3.3 \%)$ were transfused with packed red blood cells (PRBC): two patients received two units and two further patients recieved one unit in the complicated group, while one patient received one unit in the non-complicated group.

Overall median length of stay was 3 (IQR 1-10) days and did not significantly differ between the groups $(p=0.19)$, and no 30-day mortalities were observed.
Table 1 Demographics and surgical details

\begin{tabular}{lllll}
\hline & All $(n=150)$ & Complicated $(n=78)$ & $\begin{array}{l}\text { Uncom- } \\
\text { plicated } \\
(\mathrm{n}=72)\end{array}$ & $p$ \\
\hline Age (years) (mean $\pm \mathrm{SD})$ & & & $60.1 \pm 10.2$ & 0.95 \\
Gender $($ male, $\%)$ & $60.2 \pm 11.1$ & $60.2 \pm 11.8$ & $32(44.4)$ & 0.87 \\
BMI $\left(\mathrm{kg} / \mathrm{m}^{2}\right)($ mean $\pm \mathrm{SD})$ & $68(45.3)$ & $36(46.2)$ & $27.1 \pm 5.3$ & 0.05 \\
ASA $\geq 3(\%)$ & $28.2 \pm 5.4$ & $28.9 \pm 5.6$ & $30(41.7)$ & 0.42 \\
Immunosuppression $(\%)$ & $68(45.3)$ & $38(48.7)$ & $3(4.2)$ & 0.35 \\
Previous abdominal surgery $(\%)$ & $4(2.7)$ & $1(1.3)$ & $16(22.2)$ & 0.28 \\
Contamination class $\geq 3(\%)$ & $28(18.7)$ & $12(15.4)$ & $17(23.6)$ & $\mathbf{0 . 0 0 0 0 2}$ \\
Operation duration $($ minutes) $($ mean $\pm \mathrm{SD})$ & $270 \pm 78$ & $288 \pm 96$ & $258 \pm 72$ & $\mathbf{0 . 0 4}$ \\
Additional organ resection $(\%)$ & $17(11.3)$ & $10(12.8)$ & $7(9.7)$ & 0.61 \\
Anastomosis $(\%)$ & $144(96)$ & $73(93.6)$ & $71(98.6)$ & 0.21 \\
Diversion $(\%)$ & $33(22)$ & $23(29.5)$ & $10(13.9)$ & $\mathbf{0 . 0 3}$ \\
Conversion $(\%)$ & $10(6.7)$ & $8(10.3)$ & $2(2.8)$ & 0.10 \\
\hline
\end{tabular}

Baseline demographic and surgical parameters of patients with complicated diverticulitis $(n=78)$ and patients with uncomplicated diverticulitis $(n=72)$

$B M I$ body mass index, ASA American Society of Anesthesiology Score

Bold characters indicate significant values $(p<0.05)$ 
Table 2 Outcome

\begin{tabular}{lllll}
\hline & All $(n=150)$ & Complicated $(n=78)$ & $\begin{array}{l}\text { Uncompli- } \\
\text { cated }(n=72)\end{array}$ & $p$ \\
\hline Any complication (I-V) (\%) & $33(22)$ & $22(28.2)$ & $11(15.3)$ & 0.08 \\
Major complication (IIIb-V) (\%) & $7(4.7)$ & $5(6.4)$ & $2(2.8)$ & 0.45 \\
Minor complication (I-IIIa) (\%) & $30(20)$ & $20(25.6)$ & $10(13.9)$ & 0.10 \\
Surgical complication (\%) & $26(17.3)$ & $17(21.8)$ & $9(12.5)$ & 0.20 \\
Ileus (\%) & $12(8)$ & $8(10.3)$ & $4(5.6)$ & 0.37 \\
SBO (\%) & $2(1.3)$ & 0 & $2(2.8)$ & 0.23 \\
Anastomotic leak (\%) & $5 / 144(3.5)$ & $3 / 73(4.1)$ & $2 / 71(2.8)$ & 0.67 \\
SSI (\%) & $12(8)$ & $8(10.3)$ & $4(5.6)$ & 0.29 \\
Bleeding complication (\%) & $5(3.3)$ & $4(5.1)$ & $1(1.4)$ & 0.37 \\
Postoperative anemia (\%) & $6(4)$ & $6(7.7)$ & 0 & $\mathbf{0 . 0 3}$ \\
Postoperative transfusion (\%) & $4(2.7)$ & $3(3.8)$ & $1(1.4)$ & 0.62 \\
Hemoglobin POD 1 (g/L) & $11.9 \pm 1.5$ & $11.6 \pm 1.6$ & $12.2 \pm 1.4$ & $\mathbf{0 . 0 2}$ \\
Urinary retention (\%) & $10(6.7)$ & $5(6.4)$ & $5(6.9)$ & 1.00 \\
Medical complication (\%) & $18(12)$ & $12(15.4)$ & $6(8.3)$ & 0.22 \\
Reoperation (\%) & $4(2.7)$ & $2(2.6)$ & $2(2.8)$ & 1.00 \\
Readmission (\%) & $11(7.3)$ & $6(7.7)$ & $5(6.9)$ & 0.86 \\
Length of stay (days) (median, IQR) & $3(1-10)$ & $3(2-10)$ & $3(1-5)$ & 0.19 \\
\hline Posto
\end{tabular}

Postoperative complication in patients with complicated diverticulitis $(n=78)$ and patients with uncomplicated diverticulitis $(n=72)$

$S B O$ small bowel obstruction, $S S I$ surgical site infection, $P O D$ postoperative day

Bold characters indicate significant values $(p<0.05)$

\section{Discussion}

This study revealed favorable short-term outcomes and a median hospital stay of 3 days after robotic left-sided colectomy for diverticular disease. Over half of patients presented with complicated disease. Robotic surgery was shown to be feasible and safe. High compliance to the institutional ERP, regardless of disease presentation, may have contributed to these encouraging short-term results.

Whether and when symptomatic, recurrent diverticular disease needs to be managed surgically is a matter of ongoing debate $[2,14]$. Further, the ideal surgical approach has not yet been defined, even though laparoscopic surgery is presently considered the standard of care due to its potential to improve pain and recovery resulting in a decreased length of stay [15-17]. However, laparoscopic approaches may be challenging in complicated diverticular disease, as demonstrated by conversion rates of up to $14 \%$ [18, 19]. The first institutional series (1993-2004) reporting on both, complicated (37\%) and uncomplicated (63\%) diverticulitis, revealed a conversion rate of $26 \%$ and a 30-day overall complication rate of $25 \%$ [20]. A further institutional experience, which specifically assessed perioperative care within a fast track protocol, revealed an overall morbidity rate of $25.5 \%$ in conventional care and $15.2 \%$ in fast track care, which was also associated with improved gastrointestinal recovery [21].
The robotic platform represents a more recent treatment alternative for diverticular surgery and was associated with favorable outcomes in small retrospective case series. Maciel et al. described no conversions in 20 robotic procedures for fistulizing diverticular disease, compared to a rate of $14.5 \%$ in the laparoscopic comparative group [22]. A pioneer study reporting on 24 patients undergoing robotic resection 10 years ago showed encouraging results for recurrent diverticular disease [23]. Furthermore, recent reports demonstrated robotic resection of diverticular disease to be safe and technically feasible, however, within a case mix of oncological and non-oncological indications [24, 25].

The present study reports on 150 patients operated on in a center with advanced experience in robotic surgery. Conversion was slightly lower (6.7\%) compared to reports in similar settings [26], and Hartmann procedures could be avoided in $96 \%$ of patients. The most recent institutional series of 361 patients with diverticular disease (38\% complicated) operated by a minimally invasive approach found a conversion rate of $14 \%$ in complicated and $11.6 \%$ in uncomplicated disease, while overall morbidity was similar to the present study [19]. Even though the leak rate of $2 \%$ was slightly lower in this former series, length of stay averaged 5 days, with one-third of patients treated within a fast track protocol. Implementation of the institutional ERP in 2010 lead to decreased length of stay with a median of 3 days [21], as seen in the present study. 
The most important finding of our study is the similarity in complication rates and length of stay regardless of disease presentation by the time of surgery. Several factors may contribute to this result. First, advanced expertise in robotic surgery allowed for safe surgery despite unfavorable and challenging conditions with complicated disease. Second, standardized approach and teaching conditions lead to better outcomes, as repeatedly shown previously [27-29]. Finally and most importantly, high compliance to the institutional ERP, yielding over $70 \%$ even in patients with complicated disease, may have significantly contributed to these encouraging results. High ERP compliance regardless of the surgical approach has been repeatedly associated with favorable short-term outcomes [30, 31].

The present study has limitations that need to be addressed. The limited number of patients in both groups is prone to type II-error (small sample size). However, this study reports on the largest cohort of robotically performed colectomies for complicated diverticular disease. Since not all consecutive patients operated on for diverticular disease were performed robotically, selection bias may exist. However, over half of patients presented with complicated disease at the time of surgery, rendering a positive selection bias unlikely. Finally, the present results derive from an expert center and cannot be uncritically extrapolated to other settings.

In conclusion, elective robotic resection of complicated left-sided diverticular disease was feasible and safe in this series with encouraging short-term results regardless of disease presentation by the time of surgery. Surgical expertise, standardization and high ERP adherence may be key factors.

Acknowledgements Fabian Grass was supported by the Société Académique Vaudoise, Lausanne, Switzerland and by the SICPA foundation, Lausanne, Switzerland.

Funding No funding sources to declare.

\section{Compliance with ethical standards}

Disclosures Drs. Fabian Grass, Jacopo Crippa, Kellie L. Mathis, Scott R. Kelley, David W. Larson have no conflicts of interest or financial ties to disclose.

\section{References}

1. Munie ST, Nalamati SPM (2018) Epidemiology and pathophysiology of diverticular disease. Clin Colon Rectal Surg 31:209-213

2. van de Wall BJM, Stam MAW, Draaisma WA, Stellato R, Bemelman WA, Boermeester MA, Broeders I, Belgers EJ, Toorenvliet BR, Prins HA, Consten ECJ (2017) Surgery versus conservative management for recurrent and ongoing left-sided diverticulitis (DIRECT trial): an open-label, multicentre, randomised controlled trial. Lancet Gastroenterol Hepatol 2:13-22
3. Van Arendonk KJ, Tymitz KM, Gearhart SL, Stem M, Lidor AO (2013) Outcomes and costs of elective surgery for diverticular disease: a comparison with other diseases requiring colectomy. JAMA Surg 148:316-321

4. Halabi WJ, Kang CY, Jafari MD, Nguyen VQ, Carmichael JC, Mills S, Stamos MJ, Pigazzi A (2013) Robotic-assisted colorectal surgery in the United States: a nationwide analysis of trends and outcomes. World J Surg 37:2782-2790

5. Jayne D, Pigazzi A, Marshall H, Croft J, Corrigan N, Copeland J, Quirke P, West N, Rautio T, Thomassen N, Tilney H, Gudgeon M, Bianchi PP, Edlin R, Hulme C, Brown J (2017) Effect of robotic-assisted vs conventional laparoscopic surgery on risk of conversion to open laparotomy among patients undergoing resection for rectal cancer: the ROLARR randomized clinical trial. JAMA 318:1569-1580

6. Lorenzon L, Bini F, Balducci G, Ferri M, Salvi PF, Marinozzi F (2016) Laparoscopic versus robotic-assisted colectomy and rectal resection: a systematic review and meta-analysis. Int $\mathrm{J}$ Colorectal Dis 31:161-173

7. Duchalais E, Machairas N, Kelley SR, Landmann RG, Merchea A, Colibaseanu DT, Mathis KL, Dozois EJ, Larson DW (2018) Does prolonged operative time impact postoperative morbidity in patients undergoing robotic-assisted rectal resection for cancer? Surg Endosc 32:3659-3666

8. Kelley SR, Duchalais E, Larson DW (2018) Robotic right colectomy with intracorporeal anastomosis for malignancy. J Robot Surg 12:461-466

9. Lightner AL, Kelley SR, Larson DW (2018) Robotic Platform for an IPAA. Dis Colon Rectum 61:869-874

10. Boostrom SY, Wolff BG, Cima RR, Merchea A, Dozois EJ, Larson DW (2012) Uncomplicated diverticulitis, more complicated than we thought. J Gastrointest Surg 16:1744-1749

11. Emori TG, Culver DH, Horan TC, Jarvis WR, White JW, Olson DR, Banerjee S, Edwards JR, Martone WJ, Gaynes RP et al (1991) National nosocomial infections surveillance system (NNIS): description of surveillance methods. Am J Infect Control 19:19-35

12. Larson DW, Lovely JK, Cima RR, Dozois EJ, Chua H, Wolff BG, Pemberton JH, Devine RR, Huebner M (2014) Outcomes after implementation of a multimodal standard care pathway for laparoscopic colorectal surgery. Br J Surg 101:1023-1030

13. Dindo D, Demartines N, Clavien PA (2004) Classification of surgical complications: a new proposal with evaluation in a cohort of 6336 patients and results of a survey. Ann Surg 240:205-213

14. Khan RMA, Hajibandeh S, Hajibandeh S (2017) Early elective versus delayed elective surgery in acute recurrent diverticulitis: a systematic review and meta-analysis. Int J Surg 46:92-101

15. Abraha I, Binda GA, Montedori A, Arezzo A, Cirocchi R (2017) Laparoscopic versus open resection for sigmoid diverticulitis. Cochrane Database Syst Rev 11::CD009277

16. Gralista P, Moris D, Vailas M, Angelou A, Petrou A, Felekouras E, Papalampros A (2017) Laparoscopic approach in colonic diverticulitis: dispelling myths and misperceptions. Surg Laparosc Endosc Percutan Tech 27:73-82

17. Cirocchi R, Arezzo A, Renzi C, Cochetti G, D’Andrea V, Fingerhut A, Mearini E, Binda GA (2015) Is laparoscopic surgery the best treatment in fistulas complicating diverticular disease of the sigmoid colon? A systematic review. Int J Surg 24:95-100

18. Bhakta A, Tafen M, Glotzer O, Canete J, Chismark AD, Valerian BT, Stain SC, Lee EC (2016) Laparoscopic sigmoid colectomy for complicated diverticulitis is safe: review of 576 consecutive colectomies. Surg Endosc 30:1629-1634

19. Pendlimari R, Touzios JG, Azodo IA, Chua HK, Dozois EJ, Cima RR, Larson DW (2011) Short-term outcomes after elective minimally invasive colectomy for diverticulitis. Br J Surg 98:431-435 
20. Hassan I, Cima RR, Larson DW, Dozois EJ, O’Byrne MM, Larson DR, Pemberton JH (2007) The impact of uncomplicated and complicated diverticulitis on laparoscopic surgery conversion rates and patient outcomes. Surg Endosc 21:1690-1694

21. Larson DW, Batdorf NJ, Touzios JG, Cima RR, Chua HK, Pemberton JH, Dozois EJ (2010) A fast-track recovery protocol improves outcomes in elective laparoscopic colectomy for diverticulitis. J Am Coll Surg 211:485-489

22. Maciel V, Lujan HJ, Plasencia G, Zeichen M, Mata W, Jorge I, Lee D, 3rd Viamonte M, Hartmann RF (2014) Diverticular disease complicated with colovesical fistula: laparoscopic versus robotic management. Int Surg 99:203-210

23. Ragupathi M, Ramos-Valadez DI, Patel CB, Haas EM (2011) Robotic-assisted laparoscopic surgery for recurrent diverticulitis: experience in consecutive cases and a review of the literature. Surg Endosc 25:199-206

24. Spinelli A, David G, Gidaro S, Carvello M, Sacchi M, Montorsi M, Montroni I (2017) First experience in colorectal surgery with a new robotic platform with haptic feedback. Colorectal Dis 20(3):228-235

25. Ben Salem MO, Mosbahi O, Khalgui M, Jlalia Z, Frey G, Smida M (2017) BROMETH: methodology to design safe reconfigurable medical robotic systems. Int J Med Robot 13

26. Huettner F, Pacheco PE, Doubet JL, Ryan MJ, Dynda DI, Crawford DL (2011) One hundred and two consecutive robotic-assisted minimally invasive colectomies-an outcome and technical update. J Gastrointest Surg 15:1195-1204
27. Pache B, Grass F, Fournier N, Hubner M, Demartines N, Hahnloser D (2018) Surgical teaching does not increase the risk of intraoperative adverse events. Int J Colorectal Dis

28. Page AJ, Gani F, Crowley KT, Lee KH, Grant MC, Zavadsky TL, Hobson D, Wu C, Wick EC, Pawlik TM (2016) Patient outcomes and provider perceptions following implementation of a standardized perioperative care pathway for open liver resection. Br J Surg 103:564-571

29. Collins D, Machairas N, Duchalais E, Landmann RG, Merchea A, Colibaseanu DT, Kelley SR, Mathis KL, Dozois EJ, Larson DW (2018) Participation of colon and rectal fellows in robotic rectal cancer surgery: effect on surgical outcomes. J Surg Educ 75:465-470

30. Gustafsson UO, Hausel J, Thorell A, Ljungqvist O, Soop M, Nygren J, Enhanced Recovery After Surgery Study G (2011) Adherence to the enhanced recovery after surgery protocol and outcomes after colorectal cancer surgery. Arch Surg 146:571-577

31. Jurt J, Slieker J, Frauche P, Addor V, Sola J, Demartines N, Hubner M (2017) Enhanced recovery after surgery: can we rely on the key factors or do we need the bel ensemble? World J Surg 41:2464-2470

Publisher's Note Springer Nature remains neutral with regard to jurisdictional claims in published maps and institutional affiliations. 